\section{Ein neues Phosphorlebertranöl.}

\section{Von Dr. Hugo Korte in Hamburg.}

Die Wichtigkeit der therapeutischen Anwendung von Phosphor bei Rachitis in der Form des Phosphorlebertrans ist bekannt. Die leichte Oxydierbarkeit des Phosphors bot jedoch bisher dem Arzte keine Gewähr für ein haltbares Präparat mit vorgeschriebenem Gehalt. Dieser Mangel wird auch nicht beseitigt durch die Forderung des deutschen Arzneibuches, das Phosphorlebertranöl entsprechend der ärztlichen Verordnung jedesmal frisch zu bereiten, da es geradezu unmöglich ist, $1 \mathrm{cg}$ Phosphor genau abzuwägen, bevor es zur Entzündung kommt; und sollte es auch nicht zur Entzündung kommen, so ist ein solch kleines Stück der umgebenden Luft dermaßen ausgesetzt, daß ein großer Teil seines Gewichtes in Oxyd verwandelt wird, wodurch die genaue Dispensation ohne weiteres illusorisch gemacht ist. Es werden daher in den meisten Apotheken'Stammlösungen vorrätig gehalten, die aber schon in kurzer Zeit sich merklich verändert haben. So fand ich bei zehn den Apotheken entnommenen Proben Unterschiede von 30 bis $80 \%$ des vorgeschriebenen Phosphorgehaltes.

Um diesen Uebelständen zu begegnen und da anch von den im Jahre 1904 schon bekannten Vorschriften der Pharmakopöen verschiedener Länder keine den an sie gestellten Ansprüchen eines einwandfreien Phosphoröles gerecht wurde, ist mir seinerzeit von Herrn Geheimrat Prof. Dr. Heffter, jetzt Leiter des Pharmakologischen Instituts der Universität Berlin, die Aufgabe gestellt worden, ein Phosphoröl herzustellen, das allen vom Arzte gestellten Ansprüchen auf Haltbarkeit zu jeder Jahreszeit und konstantem Phosphorgehalt entspricht.

In zweijähriger Arbeit, bei der nahezu 400 Analysen von mir angefertigt wurden, bin ich zu einer vollkommen befriedigenden Lösung der Frage gelangt. Ich verweise auf meine Arbeit: „Philosophische Inauguraldissertation. Bern 1906. Ueber die Haltbarkeit von Phosphoröl." Es hat sich hierbei ergeben, daß die Einwirkung der in den Flaschen eingeschlossenen Luft ebenso wie Temperaturunterschiede auf die Abnahme des Phosphorgehaltes nicht von der Bedeutung sind wie die Einwirkung des Lichtes und daß die Abnahme des Phosphorgehaltes in gewissen Oelen hauptsächlich dadurch bedingt zu sein scheint, daß sich der Phosphor in eine unlösliche Modifikation oder Verbindung umwandelt.
Im Verlaufe der Arbeit habe ich Versuche über die Haltbarkeit, von Oelen angestellt, denen 5\% Alkohol zugesetzt war. Ebenso wie es Stoffe gibt, deren Gegenwart beschleunigend auf eine chemische Reaktion wirkt, so gibt es auch solche, deren Gegenwart die Geschwindigkeit gewisser Reaktionen hemmt. So ist es eine von Graham und von Centnerszwer ${ }^{1}$ ) festgestellte Tatsache, daß Spuren von Aethylen, Alkohol, Aether, Terpentinöl, Jodäthyl etc. das Leuchten, d. h. also auch die Oxydation des Phosphors verhindern.

Von einem 5\% Alkohol enthaltenden Phosphorolivenöl zeigten die im Dunkeln aufbewahrten Proben nach fünfmonatiger Aufbewahrung einen Durchschnittsgehalt von $93,4 \%$ nach weiteren drei Monaten einen solchen von 90,3\%, ein nach fünf Monaten untersuchtes, $5 \%$ Alkohol enthaltendes Phosphormandelöl 94,3\%. Es ist durch den Zusatz von Alkohol die Haltbarkeit nicht wesentlich gefördert worden.

Ich habe dann Versuche angestellt mit Phosphorölen, denen ich $1 \%$ Limonen ${ }^{2}$ ) zusetzte. Limonen ist ein Terpen von der Formel $\mathrm{C}_{10} \mathrm{H}_{18}$, das sich bekanntlich in verschiedenen Oelen, besonders den Oelen von Citrus Limonum und Citrus Aurantium Risso vorfindet. Die nach 6-7 Monaten untersuchten Oele zeigten immer noch einen Phosphorgehalt von $99-100 \%$ des ursprünglichen Phosphors. Die mit Limonenzusatz hergestellten Oele sind also sehr gut haltbar, sogar auch dann, wenn sie in hellen Gläsern aufbewahrt werden. Tatsächlich zeigte keines der Oele eine mit dem Auge wahrnehmbare Veränderung. Zur Erzielung eines haltbaren Phosphoröles ist daher seine Zubereitung unter Zusatz von Limonen unter allen Umständen eine unumgängliche Forderung. $\left.{ }^{3}\right)$

Mein Präparat "Oleum jecor. aselli phosphoratum anticatalysatum $^{*}$ wird von meiner Firma Dr. Korte \& Co., Hamburg 15 unter dem Namen "Phosrhachit" in den Verkehr gebracht in der gewöhnlichen Dosierung 0,01:100,0 zu dem Preise von 1,00 M (entsprechend einem gewöhnlichen Phosphorlebertran nach der deutschen Arzneitaxe), sodaß seine Anwendung ohne weiteres auch in der Kassenpraxis ermöglicht ist.

Proben versendet meine Fabrik frei und unberechnet. 